\title{
Optimal Interception with Time Constraint ${ }^{1}$
}

\author{
N. X. VINH, ${ }^{2}$ P. LU, ${ }^{3}$ R. M. HOWE, ${ }^{2}$ AND E. G. GILBERT ${ }^{2}$
}

Communicated by A. Miele

\begin{abstract}
This paper considers the problem of minimum-fuel interception with time constraint. The maneuver consists of using impulsive thrust to bring the interceptor from its initial orbit into a collision course with a target which is moving on a well-defined trajectory. The intercept time is either prescribed or is restricted to be less than an upper limit.

The necessary conditions and the transversality conditions for optimality are discussed. The method of solution amounts to first solving a set of equations to obtain the primer vector for an initial one-impulse solution. Then, based on the information provided by the primer vector, rules are established to search for the optimal solution if the initial one-impulse trajectory is not optimal. The method is general, in the sense that it allows for solving the problem of three-dimensional interception with arbitrary motion for the target.

Several numerical examples are presented, including orbital interceptions and interception at hyperbolic speeds of a ballistic missile.
\end{abstract}

Key Words. Orbital transfer, optimal interception, primer vector theory, hodograph theory, Lambert's problem, interception of ballistic missiles.

\section{Introduction}

The problems of interception and rendezvous with time constraint are two fundamental problems in space maneuvering. We shall consider the first problem in this paper. In the following content, boldface notations represent vectors; plainface notations stand for the magnitudes of the

\footnotetext{
${ }^{1}$ This research was supported by US Army Strategic Defense Command, Contract No. DASG60-88-C-0037.

${ }^{2}$ Professor of Aerospace Engineering, University of Michigan, Ann Arbor, Michigan.

${ }^{3}$ Research Associate, University of Michigan, Ann Arbor, Michigan.
} 
corresponding vectors and scalar variables. The product of two vectors is understood as inner product.

The interceptor is initially in a motion defined by its position vector $\mathbf{r}_{0}(t)$ assumed known. At a certain time $t_{0}$, called acquisition time or sometimes initial time, the target is at the position $\mathbf{r}_{T}\left(t_{0}\right)$ with velocity $\mathbf{V}_{T}\left(t_{0}\right)$, assumed known. Hence, if its subsequent motion is uncontrolled and is subject only to a Newtonian gravitational attraction, it is well determined by the two functions $\mathbf{r}_{T}(t)$ and $\mathbf{V}_{T}(t)$ which can be computed from the given data. It is proposed to intercept the target at a final time $t_{f}>t_{0}$ so that the characteristic velocity required for the transfer is minimum. The specific assumption on $t_{0}$ and $t_{f}$ will be given later when we consider the different types of interception.

It should be noticed that, for the sake of generality, the function $\mathbf{r}_{0}(t)$ can be completely arbitrary. It may represent an orbital Keplerian motion for the interceptor or an atmospheric ascent trajectory for a rocket or an airplane which carries the interceptor. Likewise, we can simply assume that the function $\mathbf{r}_{T}(t)$ defining the motion of the target is known. On the other hand, we shall assume that, in the time interval $\left[t_{1}, t_{f}\right]$, where the time $t_{1}$, $t_{0} \leq t_{1}<t_{f}$, is the instant of the first ignition of the control engine, the interceptor is subject only to the inverse-square force field and a controlled action of a propulsive force $\mathbf{F}$.

\section{Necessary Conditions for Optimality}

We consider the general problem of transfer. A rocket, considered as a mass point with varying mass, is governed by the equations

$$
\begin{aligned}
& \dot{\mathbf{r}}=\mathbf{V}, \\
& \dot{\mathbf{V}}=\mathbf{g}+\boldsymbol{\Gamma}, \\
& \dot{U}=\Gamma,
\end{aligned}
$$

where $\mathbf{g}$ is the acceleration of gravity, a function of the position, and $U$ is the characteristic velocity spent since the initial time,

$$
U=\int_{t_{0}}^{t} \Gamma d t
$$

with $\mathbf{\Gamma}=\mathbf{F} / m$ being the thrust acceleration. For a high-thrust propulsion system, $U$ is a measure of the fuel consumption.

Consider the Hamiltonian of the system,

$$
H=\mathbf{p}_{r} \mathbf{V}+\mathbf{p}_{v}(\boldsymbol{\Gamma}+\mathbf{g})+p_{U} \Gamma,
$$


where the adjoint variables $\mathbf{p}_{r}, \mathbf{p}_{v}$, and $p_{U}$ satisfy the equations

$$
\begin{aligned}
& \dot{\mathbf{p}}_{r}=-\partial H / \partial \mathbf{r}, \\
& \dot{\mathbf{p}}_{V}=-\partial H / \partial \mathbf{V}=-\mathbf{p}_{r}, \\
& \dot{p}_{U}=-\partial H / \partial U .
\end{aligned}
$$

To maximize $H$ with respect to the control vector $\Gamma$, we first maximize the product $\mathbf{p}_{V} \boldsymbol{\Gamma}$. Then, $\boldsymbol{\Gamma}$ must be selected parallel to $\mathbf{p}_{V}$, and hence

$$
H^{*}=\mathbf{p}_{r} \mathbf{V}+\mathbf{p}_{V} \mathbf{g}+\left(p_{V}+p_{U}\right) \Gamma,
$$

where $p_{v}$ is the length of $\mathbf{p}_{v}$.

The thrust acceleration is now linear, subject to

$$
0 \leq \Gamma \leq \Gamma_{\max } \text {. }
$$

Consider the switching function

$$
\kappa=p_{V}+p_{U} \text {. }
$$

Then,

$$
\begin{aligned}
& \text { if } \kappa>0, \text { select } \Gamma=\Gamma_{\max } \text { (boost arc); } \\
& \text { if } \kappa<0 \text {, select } \Gamma=0 \text { (coast arc); } \\
& \text { if } \kappa \equiv 0 \text {, select } \Gamma=\text { variable (sustained arc). }
\end{aligned}
$$

We have Lawden's optimal law for the thrust control (Refs. 1 and 2): (i) whenever the engine is operating, the thrust direction is parallel to the vector $\mathbf{p}_{v}$, called the primer vector; and (ii) if $\kappa>0$, we use $\Gamma=\Gamma_{\max }$; if $\kappa<0$, we use $\Gamma=0$; the thrust is switched on and off at $\kappa=0$.

The problem is solved if we know the time history of $\mathbf{p}_{V}$ and the switching function $\kappa$. For example, if we plot the function $\kappa$ versus the time, we have the typical variation shown in Fig. 1. We use the maximum thrust directed along $\mathbf{p}_{V}$ between $t_{1}$ and $t_{2}$ and then between $t_{3}$ and $t_{4}$. The remaining arcs are coast arcs. Of course, the terminal conditions must be

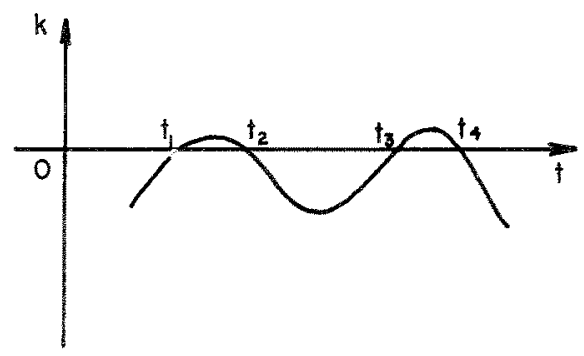

Fig. 1. Switching function for finite thrust. 
satisfied. For very high thrust, we can use the approximation $\Gamma_{\max } \rightarrow \infty$. The time interval $\Delta t$ for each boost arc tends to zero, and we shall have the typical variation of $\kappa$ in Fig. 2. The thrusting phases are approximated by the impulses $I_{1}$ and $I_{2}$. For impulsive thrust, by changing the independent variable from $t$ to $U$, it can be shown that, across an impulse, the functions $\mathbf{r}(t), \mathbf{g}(\mathbf{r}), \mathbf{p}_{r}$, and $\mathbf{p}_{V}$ are continuous (Ref. 2). On the other hand, we write

$$
d \mathbf{V} / d U=\mathbf{g} / \Gamma_{\max }+\mathbf{p}_{V} / p_{V} \rightarrow \mathbf{p}_{V} / p_{V}
$$

Hence, integrating across an impulse, we have a discontinuity in $\mathrm{V}$,

$$
\Delta \mathbf{V}=\mathbf{V}_{2}-\mathbf{V}_{1}=\left(\mathbf{p}_{V} / p_{V}\right) \Delta V
$$

where $\Delta V$ is the characteristic velocity change across an impulse.

The adjoint $p_{U}$ satisfies the equation

$$
\dot{p}_{U}=-\partial H^{*} / \partial U=-(\partial / \partial U)\left[\Gamma^{*} \kappa\right] .
$$

On a coast arc, $\Gamma^{*}=0$; on a sustained arc, $\kappa=0$, and we have $p_{U}=$ const. But on a boost arc, with $\Gamma^{*}=\Gamma_{\max }(U)$, we consider the equation for the variation of the mass,

$$
d m / d t=-F_{\max } / c=-m \Gamma_{\max } / c,
$$

where $c$ is the constant exhaust velocity for a high-thrust propulsion system. By integrating the equation, we obtain

$$
m=m_{0} \exp (-U / c) \text {. }
$$

Hence,

$$
\Gamma_{\max }(U)=F_{\max } / m=\left(F_{\max } / m_{0}\right) \exp (U / c) .
$$

The adjoint equation for $p_{U}$ becomes

$$
\dot{p}_{U}=-\Gamma_{\max } \kappa / c .
$$

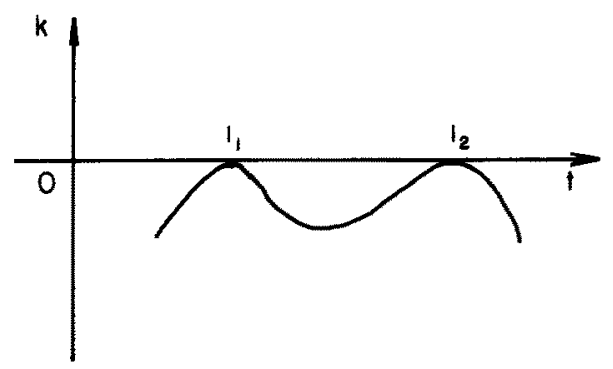

Fig. 2. Switching function for impulsive thrust. 
Since $\kappa>0$ on a boost arc, $p_{U}$ is decreasing along a boost arc. For the case of infinite thrust, since across an impulse $U$ has a finite variation, we write

$$
d p_{U} / d U=-\kappa / c .
$$

Across an impulse, $\kappa=0$; hence, we also have $p_{U}=$ const. We conclude that, in the impulsive case, in the closed interval $\left[t_{0}, t_{f}\right], p_{U}=$ const; and, from the transversality condition in the next section,

$$
p_{U}=p_{U f}=-1 \text {. }
$$

It is then sufficient to consider $\mathbf{p}_{V}$ on coast arcs for impulsive thrust, since we have $\kappa=p_{V}+p_{U} \leq 0$; therefore, $p_{V} \leq 1$ on the interval $\left[t_{0}, t_{f}\right]$.

The solution for $\mathbf{p}_{V}$ on a coasting arc has been obtained by Lawden for a Newtonian central force field in Ref. 1 and by Vinh for a general, time-invariant force field in Ref. 3.

Along a coasting arc, we consider a rotating coordinate system MSTW with $M$ at the rocket, the $S$-axis along the position vector, positive outward, the $T$-axis in the plane of the motion, orthogonal to the $S$-axis and positive in the direction of motion, and the $W$-axis completing a right-handed system as shown in Fig. 3. Notice that $\theta$ is the true anomaly measured from the perigee of the osculating orbit.

Let $S, T$, and $W$ be the components of the primer vector $\mathbf{p}_{V}$. We have $(\operatorname{Ref}, 1)$

$$
\begin{aligned}
S & =A \cos \theta+B e \sin \theta+C I_{1}, \\
T & =-A \sin \theta+B(1+e \cos \theta) \\
& +(D-A \sin \theta) /(1+e \cos \theta)+C I_{2}, \\
W & =(E \cos \theta+F \sin \theta) /(1+e \cos \theta),
\end{aligned}
$$

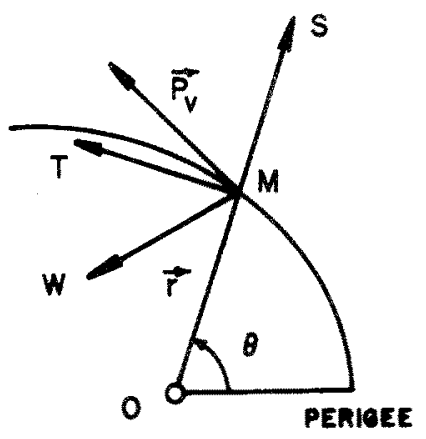

Fig. 3. Rotating coordinate system. 
where

$$
\begin{aligned}
I_{1} & =\left[1 /\left(1-e^{2}\right)\right][-\cos \theta+2 e /(1+e \cos \theta) \\
& \left.-3 \sqrt{\left(\mu / p^{3}\right)} t e^{2} \sin \theta\right], \\
I_{2} & =r \cos \theta / e p \sin \theta+(p / e r \sin \theta) I_{1} .
\end{aligned}
$$

Here, $\mu$ is the gravitational constant and $e$ is the eccentricity of the ballistic conic with semimajor axis $a$ and semilatus rectum $p$,

$$
\begin{array}{ll}
p=a\left(1-e^{2}\right), & \text { elliptic case } \\
p=a\left(e^{2}-1\right), & \text { hyperbolic case. }
\end{array}
$$

It is important to notice that, in Eq. (9), $t$ is the time since the passage of the perigee. The coefficients $A, B, C, D, E$, and $F$ are constants of integration to be determined.

For the analysis, we also need the components of the adjoint vector $\mathbf{p}_{r}=-\dot{\mathbf{p}}_{v}$. On the rotating axes, we have the components of the derivative of $\mathbf{p}_{v}$,

$$
\begin{aligned}
& \dot{S}=\left[\sqrt{(\mu p)} / r^{2}\right]\left[(A \sin \theta-D) /(1+e \cos \theta)-B+C I_{3}\right], \\
& \dot{T}=\sqrt{\left(\mu / p^{3}\right)}[-A(e+\cos \theta)+D e \sin \theta+C \cos \theta], \\
& \dot{W}=\sqrt{\left(\mu / p^{3}\right)}[F(e+\cos \theta)-E \sin \theta],
\end{aligned}
$$

where

$$
I_{3}=\left(e \sin ^{2} \theta-\cos \theta\right) /\left[e \sin \theta(1+e \cos \theta)^{2}\right]-I_{1} / e \sin \theta .
$$

We conclude this section with a clarification on the constant $C$. In a time-invariant force field of attraction, the Hamiltonian is constant, and we write Eq. (5) as follows:

$$
H^{*}=\mathbf{p}_{r} \mathbf{V}+\mathbf{p}_{V} \mathbf{g}+\Gamma^{*} \kappa=\bar{C} .
$$

Since the equation is valid over the whole optimal trajectory, it suffices to evaluate the constant on a coast arc with $\Gamma^{*}=0$. Lawden's solutions for $\mathbf{p}_{V}$ and $\mathbf{p}_{r}=-\dot{\mathbf{p}}_{v}$ as given in Eqs. (8) and (11) apply separately for each coast arc, with $\theta=0$ and $t=0$ at the perigee of the transfer orbit. When connecting several arcs by impulses, the constants of integration $A, B, \ldots, F$ and time $t$ have to be adjusted accordingly. In particular, using Eqs. (8) and (11) for $\mathbf{p}_{V}$ and $\mathbf{p}_{r}$, with the components on the MSTW system

$$
\begin{aligned}
& \mathbf{V}=(\sqrt{(\mu / p)} e \sin \theta, \sqrt{(\mu p)} / r, 0), \\
& \mathbf{g}=\left(-\mu / r^{2}, 0,0\right),
\end{aligned}
$$


by substituting the above equations and (8) and (11) into the Hamiltonian, we arrive at

$$
-\left(\mu e / p^{2}\right) C=\bar{C},
$$

where $\bar{C}=H^{*}$ is the global constant, and $C$ is the constant on each coasting arc with eccentricity $e$ and semilatus rectum $p$. For this reason, whenever $H^{*}=0$, we simply take $C=0$.

Across an impulse, the term $\Gamma^{*} \mathrm{~K}$ has the indeterminate form $\infty \times 0$. But this term is zero before and after an interior impulse, since we have then either a coasting arc or a sustained arc. Hence, since $\mathbf{p}_{r}$ and $\mathbf{p}_{V}$ are continuous across an impulse,

$$
H^{*-}=\mathbf{p}_{r} \mathbf{V}^{-}+\mathbf{p}_{v} \mathbf{g}=\mathbf{p}_{r} \mathbf{V}^{+}+\mathbf{p}_{v} \mathbf{g}=\mathrm{H}^{*+} .
$$

Because $\Gamma^{*}$ is parallel to $\mathbf{p}_{V}$, so is $\Delta \mathbf{V}$; hence,

$$
\mathbf{V}^{+}=\mathbf{V}^{-}+\left(\mathbf{p}_{V} / p_{V}\right) \Delta V \text {. }
$$

From the two equations above, we obtain

$$
\mathbf{p}_{r} \mathbf{p}_{v}=0 \text {. }
$$

Hence,

$$
p_{V} \dot{p}_{V}=\mathbf{p}_{V} \dot{\mathbf{p}}_{V}=-\mathbf{p}_{V} \mathbf{p}_{r}=0 .
$$

It follows that

$$
\kappa=\dot{\kappa}=0
$$

for an interior impulse as shown in Fig. 2. As pointed out by Lawden, this is not necessarily true at the end points $t_{0}$ and $t_{f}$ if an impulse occurs there. This leads to the fact that

$$
\Gamma^{*} \kappa=0
$$

for an interior impulse and

$$
H^{*}=\mathbf{p}_{r} \mathbf{V}+\mathbf{p}_{v} \mathbf{g}
$$

in the entire open interval $\left(t_{0}, t_{f}\right)$.

\section{Transversality Conditions}

In general, the acquisition time and states as well as the final time and states may be constrained to satisfy a certain relation of the vector form

$$
\boldsymbol{\Omega}\left(\mathbf{r}_{0}, \mathbf{V}_{0}, t_{0}, \mathbf{r}_{f}, \mathbf{V}_{f}, t_{f}\right)=0 .
$$


For instance, in the interception problem one of the equations in (13) will be

$$
\mathbf{r}_{f}=\mathbf{r}_{T}\left(t_{f}\right)
$$

Equation (13) leads to a number of transversality conditions which must be satisfied by the states and the adjoint variables at the endpoints.

In the following application of the maximum principle, we adopt the Pontryagin-Contensou convention of maximizing the Hamiltonian for a minimum of the characteristic velocity. Hence, the performance index to minimize is $J=U_{f}$, which is equivalent to the maximization of the final mass for an impulsive propulsion system. Let $I$ be the augmented function

$$
I=J+\int_{t_{0}}^{t_{f}}\left(\mathbf{p}_{r} \dot{\mathbf{r}}+\mathbf{p}_{V} \dot{\mathbf{V}}+p_{U} \dot{U}-H\right) d t
$$

where $H$ is defined by (3). Then, besides the necessary conditions in Section 2 , for a stationary value of $I$, we must have the variation

$$
\delta I=\delta J+\left(\mathbf{p}_{r} \delta \mathbf{r}+\mathbf{p}_{V} \delta \mathbf{V}+p_{U} \delta U-H \delta t\right)_{0}^{f}=0
$$

with all the variations satisfying $\delta \Omega=0$. This is called the transversality condition. The constraint imposed at the endpoints in the form (13) renders the problem more difficult, more challenging to solve. We shall examine some realistic and practical situations.

First, since $U_{f}$ is arbitrary, $\delta U_{f}$ in (16) is arbitrary and independent. We have

$$
p_{U f}=-1, \quad \text { for } \delta I+p_{U f} \delta U_{f}=\left(1+p_{U f}\right) \delta U_{f}=0 .
$$

The transversality condition is reduced to

$$
\delta I=\left(\mathbf{p}_{r} \delta \mathbf{r}+\mathbf{p}_{v} \delta \mathbf{V}-H \delta t\right)_{0}^{f}=0 .
$$

Following Ref. 2, with some modification, we render explicit (17) in the following cases of interest.

Since for an interception problem the final velocity $\mathbf{V}_{f}$ is arbitrary, we have the condition $\mathbf{p}_{v f}=0$. The last arc is a coasting arc; hence, $\Gamma^{*}\left(t_{f}\right)=0$. Consequently,

$$
H_{f}^{*}=\mathbf{p}_{v f} \mathbf{V}_{j}
$$

If the initial time $t_{0}, \mathbf{r}_{0}$, and $\mathbf{V}_{0}$ are fixed, Eq. (17) becomes

$$
\mathbf{p}_{r f} \delta \mathbf{r}_{f}-H_{f}^{*} \delta t_{f}=0 \text {. }
$$

The condition is trivial if $t_{f}$ is fixed. If $t_{f}$ is not prescribed, the constraint (14) requires that $\delta \mathbf{r}_{f}=\mathbf{V}_{\gamma f} \delta t_{f}$ and we have the orthogonality condition

$$
\mathbf{p}_{r f}\left(\mathbf{V}_{T f}-\mathbf{V}_{f}\right)=0 \text {. }
$$


It should be noted that, from (18) and (19), the Hamiltonian is not necessarily equal to zero because of (14), even if $t_{f}$ is free in this case.

Another practical constraint is that $t_{0} \leq t_{f} \leq T$, for some $T>t_{0}$, with $t_{0}, r_{0}$ and $\mathbf{V}_{0}$ specified. If an optimal $t_{f}^{*}<T$ can be found, (19) remains valid. Otherwise, $t_{f}^{*}=T$; the transversality condition is modified as (Ref. 4)

$$
\mathbf{p}_{r f}\left(\mathbf{V}_{T f}-\mathbf{V}_{f}\right)=\alpha,
$$

with $\alpha$ being a multiplier.

In the case where $t_{0}$ is free and no impulse occurs at $t_{0}$,

$$
\delta \mathbf{r}_{0}=\mathbf{V}_{0}^{-} \delta t_{0}, \quad \delta \mathbf{V}_{0}=\mathbf{g}_{0} \delta t_{0} ;
$$

the transversality condition is simply

$$
H_{0}^{*}=\mathbf{p}_{r 0} \mathbf{V}_{0}^{-}+\mathbf{p}_{V_{0}} \mathbf{g}_{0} .
$$

Suppose that an optimal $t_{0}^{*}$ is found and the first impulse on the optimal trajectory occurs at $t_{1}>t_{0}^{*}$. The impulse is an interior one; therefore,

$$
\mathbf{p}_{r}\left(t_{1}\right) \mathbf{p}_{v}\left(t_{1}\right)=0 .
$$

Apparently, any $t \leq t_{1}$ (in particular, $t_{1}$ ) can also be taken as optimal initial time, because $\left[t_{0}, t_{1}\right]$ is a coast period. On the other hand, if $t_{0}^{*}=t_{1}$, any $t<t_{1}$ can also be the optimal initial time, provided that the optimal control for the interval $\left[t, t_{1}\right)$ is taken as zero. In conclusion, when $t_{0}$ is free, we can always take $t_{0}^{*}$ as the point where the first impulse occurs, and accordingly $\mathbf{p}_{r}\left(t_{0}^{*}\right) \mathbf{p}_{v}\left(t_{0}^{*}\right)=0$. Note that, in choosing to do so, the relation

$$
\mathbf{p}_{r}\left(t_{0}^{*}\right) \mathbf{p}_{V}\left(t_{0}^{*}\right)=0
$$

replaces

$$
H_{0}^{*}=\mathbf{p}_{r 0} \mathbf{V}_{0}^{-}+\mathbf{p}_{v 0} \mathbf{g}_{0} .
$$

Finally we consider the situation where $\mathbf{r}_{f}$ is fixed but not the final time $t_{f}$. This amounts to considering the target as fixed. In this case, we have trivially $H_{f}^{*}=0$, that is, $C=0$.

\section{Method of Solution}

From the discussion in the preceding sections, we see that the primer vector $\mathbf{p}_{V}$ plays an essential role in finding the optimal solution. In this section, we shall present an analytic method for obtaining the primer vector on a one-impulse trajectory. Based on the information provided by the primer vector, we shall show, if this one-impulse trajectory is not optimal, how it can be improved to approach the optimal solution. 
We consider the general three-dimensional case. Let $t_{0}$ be the initial time. We initiate the interception by application of an initial impulse at $t_{0}$. The initial position is $\mathbf{r}_{0}=\mathbf{r}_{0}\left(t_{0}\right)$ and the initial velocity is $\mathbf{V}_{0}^{-}=\dot{\mathbf{r}}_{0}\left(t_{0}\right)$. At the final time $t_{f}$, let $\mathbf{r}_{f}=\mathbf{r}_{T}\left(t_{f}\right)$ be the final position. Notice that, for any given $t_{0}$ and $t_{f}$, we can evaluate $\mathbf{r}_{0}$ and $\mathbf{r}_{f}$, and consequently the transfer angle $\Delta$ as well as the initial velocity $\mathbf{V}_{0}^{-}$before the application of the impulse. Figure 4 displays the maneuver by one impulse changing $\mathbf{V}_{0}^{-}$into $\mathbf{V}_{0}^{+}$. All the elements are now evaluated along the transfer orbit, which is well defined after solving the associated Lambert problem. In this respect, the numerical scheme developed by Battin in Ref. 5 proves to be very efficient. Since $\mathbf{V}_{0}^{-}$ and $\mathbf{V}_{0}^{+}$are known, we can compute the required impulse $\Delta \mathbf{V}_{0}$, and hence

$$
\mathbf{p}_{V}\left(t_{0}\right)=\Delta \mathbf{V}_{0} / \Delta V_{0}
$$

Explicitly, if the initial motion of the interceptor is Keplerian, let $e_{0}$ and $p_{0}$ be the eccentricity and semilatus rectum of this orbit; and let $f_{0}$ be the true anomaly defining the position $\mathbf{r}_{0}$ as measured on this orbit. After the impulse, we have the corresponding elements $e, p$, and $\theta_{0}$ of the transfer orbit obtained by solving the Lambert problem. Let $i$ be the angle between the initial orbital plane and the plane of the transfer orbit. Then, if $u, v$, and $w$ are the components of the impulse $\Delta V_{0}$ in the $M S T W$ system attached to the transfer orbit, we have

$$
\begin{aligned}
& u=\sqrt{\mu}\left[(e / \sqrt{p}) \sin \theta_{0}-\left(e_{0} / \sqrt{p_{0}}\right) \sin f_{0}\right], \\
& v=\left(\sqrt{\mu} / r_{0}\right)\left(\sqrt{p}-\sqrt{p_{0}} \cos i\right), \\
& w=\left(\sqrt{\left(\mu p_{0}\right)} / r_{0}\right) \sin i .
\end{aligned}
$$

The characteristic velocity for the transfer is

$$
\Delta V_{0}=\sqrt{ }\left(u^{2}+v^{2}+w^{2}\right) .
$$

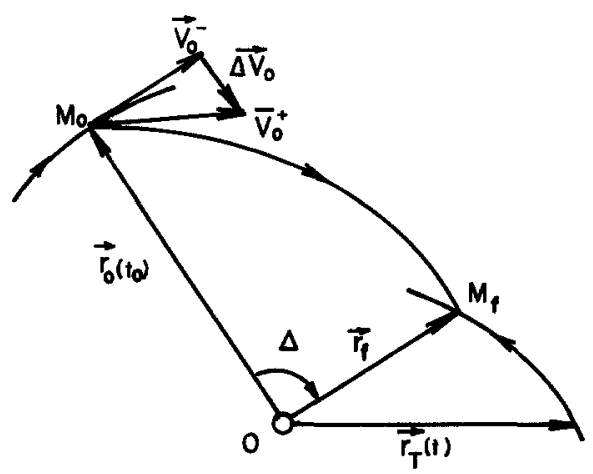

Fig. 4. Intercept trajectory. 
Since $\mathbf{p}_{V}$ is a unit vector in the direction of $\Delta \mathbf{V}$, its components $S, T$, and $W$ are its direction cosines, and we have explicitly

$$
S=u / \Delta V_{0}, \quad T=v / \Delta V_{0}, \quad W=w / \Delta V_{0} .
$$

By writing Eqs. (8) with $t=\tau_{0}$ and $\theta=\theta_{0}$, where $\tau_{0}$ is the time corresponding to $\theta_{0}$ on the transfer orbit ( $\tau=0$ when $\theta=0$ ), we have three linear equations for the six constants $A, B, \ldots, F$. For the interception problem, the final velocity is free, hence $\mathbf{p}_{v}\left(t_{f}\right)=0$ as pointed out in Section 3 . Then, by writing Eq. (8) with

$$
t=\tau_{f}=\tau_{0}+\left(t_{f}-t_{0}\right), \quad \theta=\theta_{f}=\theta_{0}+\Delta,
$$

and putting

$$
S_{f}=T_{f}=W_{f}=0,
$$

we have three more linear equations for evaluating the unknown constants. In particular, from Eq. (8c), we obtain

$$
\begin{aligned}
& E=p W \sin \theta_{f} / r_{0} \sin \Delta, \\
& F=-p W \cos \theta_{f} / r_{0} \sin \Delta .
\end{aligned}
$$

For the rest of the unknown constants, $C$ is obtained from

$$
C=N / G,
$$

where

$$
\begin{aligned}
N & =\left(p / r_{0}\right) T+S\left[e \sin \theta_{0}-2 \tan (\Delta / 2)\right], \\
G & =\left\{\left[1+e^{2}+2 e \cos \theta_{0}-2 e \sin \theta_{0} \tan (\Delta / 2)\right] /\left(e \sin \theta_{0} \sin \theta_{f}\right)\right\} \\
& \times\left[I_{1}\left(\tau_{0}\right) \sin \theta_{f}-I_{1}\left(\tau_{f}\right) \sin \theta_{0}\right]+\sin \Delta / e \sin \theta_{0} \sin \theta_{f} .
\end{aligned}
$$

$A, B$, and $D$ are then solved from the following system:

$$
\begin{aligned}
A \sin \Delta= & S \sin \theta_{f}-C\left[I_{1}\left(\tau_{0}\right) \sin \theta_{f}-I_{1}\left(\tau_{f}\right) \sin \theta_{0}\right], \\
B e \sin \Delta & =-S \cos \theta_{f}+C\left[I_{1}\left(\tau_{0}\right) \cos \theta_{f}-I_{1}\left(\tau_{f}\right) \cos \theta_{0}\right], \\
D e \sin \Delta & =-C\left\{\left[\frac{\left(2 e+\left(1+e^{2}\right) \cos \theta_{f}\right)}{\sin \theta_{f}}\right]\right. \\
& \left.\times\left[I_{1}\left(\tau_{0}\right) \sin \theta_{f}-I_{1}\left(\tau_{f}\right) \sin \theta_{0}\right]+\left(\cos \theta_{f} / \sin \theta_{f}\right) \sin \Delta\right\} \\
& +S\left[2 e+\left(1+e^{2}\right) \cos \theta_{f}\right] .
\end{aligned}
$$


With the constants evaluated, we can use Eq. (8) to calculate the magnitude of the primer vector along the transfer orbit,

$$
p_{V}(t)=\sqrt{ }\left(S^{2}+T^{2}+W^{2}\right) .
$$

In the computation, the time $t$ can be computed from Kepler's equation,

$$
\sqrt{\left(\mu / a^{3}\right)} t=E-e \sin E
$$

here, at little risk of confusion with the constant given by (24), $E$ denotes the eccentric anomaly such that

$$
\tan (\theta / 2)=\sqrt{ }[(1+e) /(1-e)] \tan (E / 2) .
$$

Once the magnitude of the primer vector is computed by the above procedure, three types of typical behaviors of $p_{V}(t)$ are plotted (see Fig. 5 ). They are representative, if not exhaustive. In the case (a), all the necessary conditions are satisfied; the one-impulse solution is thus a candidate of optimal solution. Although in the following sections we shall see that this is the case for many realistic geometrical configurations of interception and reasonable interception time $\Delta t=t_{f}-t_{0}$, it is not conclusive; so, we cannot exclude cases (b) and (c). In both of these cases, the proposed one-impulse interception is not optimal. However, the following arguments show that, for case (b), a coasting arc prior to the application of the impulse will reduce the cost; hence, the optimal solution consists of an initial coasting arc. More than one impulse are needed for the optimal solution for case (c).

First, from the calculus of variations, the first-order variation $\delta I$ of the augmented function (15) is obtained from two neighboring trajectories
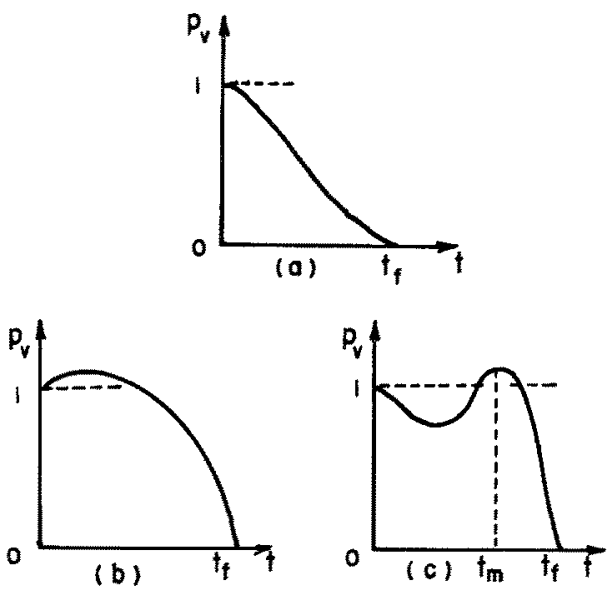

Fig. 5. Typical function $p_{V}(t)$. 
which satisfy the equations of motion (1) and the end conditions. But by (15), it is straightforward that, if the equations of motion are obeyed, the integral in (15) yields zero; thus, any variation in $I$ is a variation in $J$, namely $\delta I=\delta J$. By the expression (17), only considering the change in cost due to initial variations because they are independent of the final variations in an interception problem, we have the variation in $J$

$$
\delta J=\delta I=-\mathbf{p}_{V 0} \delta \mathbf{V}_{0}-\mathbf{p}_{r 0} \delta \mathbf{r}_{0}+H_{0}^{+} \delta t_{0},
$$

where the + sign indicates the right limits of the involved functions. Since

$$
H_{0}^{+}=\mathbf{p}_{r 0} \mathbf{V}_{0}^{+}+\mathbf{p}_{V 0} \dot{\mathbf{V}}_{0}, \quad \dot{\mathbf{V}}_{0} \delta t_{0}=\delta \mathbf{V}_{0}, \quad \delta \mathbf{r}_{0}=\mathbf{V}_{0}^{-} \delta t_{0},
$$

where $\dot{\mathbf{V}}_{0}^{+}=\dot{\mathbf{V}}_{0}^{-}$is assumed, which is generally true if $\mathbf{r}_{0}(t)$ is Keplerian motion, Eq. (33) leads to

$$
\delta J=\mathbf{p}_{r 0}\left(\mathbf{V}_{0}^{+}-\mathbf{V}_{0}^{-}\right) \delta t_{0}=\mathbf{p}_{r 0} \Delta \mathbf{V}_{0} \delta t_{0}=\Delta V_{0} \mathbf{p}_{r 0} \mathbf{p}_{v 0} \delta t_{0} .
$$

For $\delta t_{0}>0$, we see that

$$
\delta J<0, \quad \text { if }-\mathbf{p}_{r 0} \mathbf{p}_{V 0}=p_{V 0} \dot{\mathbf{p}}_{V 0}=\dot{\mathbf{p}}_{V 0}>0 .
$$

In other words, if the $p_{V}$ exceeds unity immediately after $t_{0}$ as in case (b), an initial coast will reduce the cost.

As for case (c), suppose that $T$ is the trajectory defined by $\mathbf{r}^{*}(t)$ with an initial impulse at $t_{0} ; T^{\prime}$ is a neighboring trajectory, defined by $\mathbf{r}(t)$, which passes through $\mathbf{r}^{*}\left(t_{0}\right)$ at $t_{0}$ and $\mathbf{r}^{*}\left(t_{f}\right)$ at $t_{f}$ with one initial impulse at $t_{0}$ and a midcourse impulse at some $t_{m} \in\left(t_{0}, t_{f}\right)$. According to Ref. 6 , such a trajectory can always be constructed provided a nonsingularity condition is satisfied. Along $T^{\prime}$,

$$
\begin{aligned}
& \mathbf{r}\left(t_{m}\right)=\mathbf{r}_{m}^{*}+\delta \mathbf{r}_{m}, \\
& \dot{\mathbf{r}}^{-}\left(t_{m}\right)=\dot{\mathbf{r}}_{m}^{*}+\delta \mathbf{V}_{m}^{-}, \\
& \dot{\mathbf{r}}^{+}\left(t_{m}\right)=\dot{\mathbf{r}}_{m}^{*}+\delta \mathbf{V}_{m}^{+}, \\
& \Delta \mathbf{V}_{m}=\dot{\mathbf{r}}^{+}\left(t_{m}\right)-\dot{\mathbf{r}}^{-}\left(t_{m}\right)=\delta \mathbf{V}_{m}^{+}-\delta \mathbf{V}_{m}^{-} .
\end{aligned}
$$

Also,

$$
\dot{\mathbf{r}}^{+}\left(t_{0}\right)-\dot{\mathbf{r}}^{-}\left(t_{0}\right)=\left[\dot{\mathbf{r}}^{*}\left(t_{0}\right)+\delta \mathbf{V}_{0}\right]-\dot{\mathbf{r}}^{*-}\left(t_{0}\right)=\Delta \mathbf{V}_{0}+\delta \mathbf{V}_{0},
$$

where $\delta$ stands for the small variations from $T$.

The cost on $T$ is

$$
J=\Delta V_{0}
$$

and the cost on $T^{\prime}$ is

$$
y^{\prime}=\left|\Delta \mathbf{V}_{0}+\delta \mathbf{V}_{0}\right|+\left|\delta \mathbf{V}_{m}^{+}-\delta \mathbf{V}_{m}^{-}\right|
$$


To first order, the difference is

$$
\delta J=J^{\prime}-J \approx\left(\Delta \mathbf{V}_{0} / \Delta V_{0}\right) \delta \mathbf{V}_{0}+\left|\delta \mathbf{V}_{m}^{+}-\delta \mathbf{V}_{m}^{-}\right| \text {. }
$$

Since $p_{V}\left(t_{0}\right)=\Delta \mathbf{V}_{0} / \Delta V_{0}$, we have

$$
\delta J=\mathbf{p}_{V}\left(t_{0}\right) \delta \mathbf{V}_{0}+\left|\delta \mathbf{V}_{m}^{+}-\delta \mathbf{V}_{m}^{-}\right|
$$

By a property of the adjoint variables, it is known that, along $T$,

$$
\mathbf{p}_{r} \delta \mathbf{r}+\mathbf{p}_{V} \delta \mathbf{V}=\text { const. }
$$

In particular,

$$
\begin{aligned}
& \mathbf{p}_{r}\left(t_{0}\right) \delta \mathbf{r}_{0}+\mathbf{p}_{V}\left(t_{0}\right) \delta \mathbf{V}_{0}=\mathbf{p}_{r}\left(t_{m}\right) \delta \mathbf{r}_{m}+\mathbf{p}_{V}\left(t_{m}\right) \delta \mathbf{V}_{\mathrm{m}}^{-}, \\
& \mathbf{p}_{r}\left(t_{f}\right) \delta \mathbf{r}_{f}+\mathbf{p}_{V}\left(t_{f}\right) \delta \mathbf{V}_{f}=\mathbf{p}_{r}\left(t_{m}\right) \delta \mathbf{r}_{m}+\mathbf{p}_{V}\left(t_{m}\right) \delta \mathbf{V}_{m}^{+} .
\end{aligned}
$$

Noticing that $\mathbf{p}_{V}\left(\boldsymbol{t}_{f}\right)=0$ and $\delta \mathbf{r}_{0}=\delta \mathbf{r}_{f}=0$, we add a vanishing term $-\mathbf{p}_{V}\left(t_{f}\right) \delta \mathbf{V}_{f}$ to $(35)$; and, with the aid of (36), we obtain

$$
\begin{aligned}
\delta J & =\mathbf{p}_{V}\left(t_{0}\right) \delta \mathbf{V}_{0}-\mathbf{p}_{V}\left(t_{f}\right) \delta \mathbf{V}_{f}+\left|\delta \mathbf{V}_{m}^{+}-\delta \mathbf{V}_{m}^{-}\right| \\
& =-\mathbf{p}_{V}\left(t_{m}\right)\left(\delta \mathbf{V}_{m}^{+}-\delta \mathbf{V}_{m}^{-}\right)+\left|\delta \mathbf{V}_{m}^{+}-\delta \mathbf{V}_{m}^{-}\right| \\
& =-\mathbf{p}_{V}\left(t_{m}\right) \Delta \mathbf{V}_{m}+\Delta V_{m} .
\end{aligned}
$$

Let $\mathbf{d}$ be the unit vector in the direction of $\Delta \mathbf{V}_{m}$,

$$
\delta J=\Delta V_{m}\left(1-\mathbf{p}_{V}\left(t_{m}\right) \mathbf{d}\right) .
$$

Therefore, if there exists $t_{m} \in\left(t_{0}, t_{f}\right)$ such that $p_{V}\left(t_{m}\right)>1$, a midcourse impulse can always be selected so that $\delta J<0$; the greatest descent is when $p_{V}\left(t_{m}\right)$ is maximum and $\mathbf{d}$ is in the direction $\mathbf{p}_{V}\left(t_{m}\right)$.

Note that, in Ref. 6, a proof has been given for a two-impulse trajectory, which states that, if $p_{v}>1$ between two impulses, a midcourse impulse can reduce the cost, while some modification is adopted in the above treatment for our specific objective of interception. Combining the two results, we have the rule to search for an optimal multi-impulse trajectory, if necessary, by starting with a simple one-impulse solution.

\section{Interception at Elliptic Speeds}

The necessary conditions in Section 2 and the computation of the primer vector in Section 4 are perfectly general; that is, they are applicable to a minimum-fuel three-dimensional interception problem, for any given pair of arbitrary functions $\mathbf{r}_{0}(t)$ and $\mathbf{r}_{T}(t)$ describing the initial motion of the interceptor and the motion of the target. If $\mathbf{r}_{0}(t)$ is non-Keplerian, it is sufficient to replace in Eq. (21) $\sqrt{\left(\mu / p_{0}\right)} e_{0} \sin f_{0}$ and $\sqrt{\left(\mu p_{0}\right)} / r_{0}$ by the 
components on the $S$ and $T$ axes in the initial plane of the current velocity $V_{0}^{-}$. The explicit transversality conditions derived in Section 3 , while they allow arbitrary motion of the target, specify that the initial motion of the interceptor is Keplerian. With slight modification, we can derive similar conditions for arbitrary $\mathbf{r}_{0}(t)$.

To reduce the number of parameters involved in the examples in this section, we consider the initial orbit of the interceptor as circular and take $r_{0}=1$ as the unit distance. By taking the gravitational constant $\mu=1$, the characteristic velocity is normalized with respect to the circular speed at the distance $r_{0}$. Then, $2 \pi$ is the dimensionless orbital period of the interceptor in its initial orbit. Although the dimensionless time and distance are used, to have a physical understanding of the results obtained, from time to time, we shall choose some Earth's orbits of particular altitudes in kilometers as reference to interpret.

Problem 1. The target is in an inner coplanar circular orbit at distance $r_{f}$. the initial time is preset, without loss of generality, equal to 0 . This is the same as specifying the angular distance $\omega$ at the time $t_{0}$ (Fig. 6). The final time $t_{f}$ is subject to the constraint

$$
t_{f} \leq P,
$$

where $P$ is the period of the target orbit. Alternatively, it is required to intercept the target before it completes another revolution. By the explicit transversality conditions in Section 3, if an optimal $t_{f}^{*}<P$ can be found, Eq. (19) should be met, i.e.,

$$
\mathbf{p}_{r f}\left(\mathbf{V}_{T f}-\mathbf{V}_{f}\right)=0 .
$$

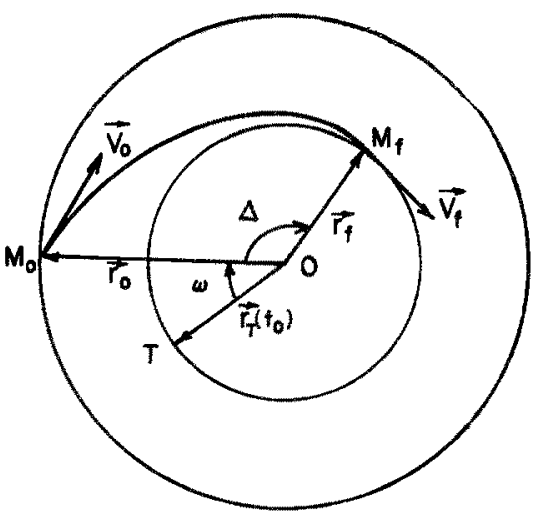

Fig. 6. Geometry of interception for Problem 1. 
Otherwise, if $t_{f}^{*}=P$, Eq. (20) holds. It should be noted that, if (38) is not present, the problem may have an optimal solution with $t_{f}^{*}>P$. As we shall see, after (38) is reinforced, the constrained optimal solution does not necessarily take $t_{f}^{*}=P$, depending on the initial lead angle $\omega$. Of course, the unconstrained optimal cost is generally better than the constrained optimal cost.

To solve this problem, we apply the technique discussed in Section 4. An initial impulse is to be applied at $t_{0}$. We take the transfer angle $\Delta$ between $\mathbf{r}_{0}$ and a trial $\mathbf{r}_{f}$ as parameter. For any given $\Delta$, we can compute the time of flight from

$$
\sqrt{\left(\mu / r_{f}\right)} t_{f}=r_{f}(\omega+\Delta) .
$$

After the associated Lambert problem is solved, the direction and the magnitude of the corresponding initial impulse are known. We can evaluate the constants in (8) by the method presented in Section 4 and then compute $\mathbf{p}_{V}$ and $\mathbf{p}_{r}$ by (8) and (11). To find a solution with $t_{f}^{*}<P,(39)$ is used for iteration to determine the correct $\Delta$, and hence $t_{f}$ from (40). Although all transversality conditions $p_{V}\left(t_{f}\right)=0$ and (39) are satisfied, for the solution to be optimal $p_{V}(t)$ must be of case (a) in Fig. 5. Figures $7(\mathrm{a})$ and $7(\mathrm{~b})$ show $p_{V}(t)$ for $\omega=20^{\circ}$ and $34.78^{\circ}$; and Fig. 7(c) shows $p_{V}(t)$ for $\omega=50^{\circ}$ (solid line), with $r_{0} / r_{f}=1.25$. For the first two values of $\omega$, the one-impulse solution is optimal and the optimal launch time coincides with $t_{0}=0$. Notice that $\dot{p}_{V}(0)=0$ for $\omega=34.78^{\circ}$. The corresponding $\Delta$ angles are $225.76^{\circ}$ and
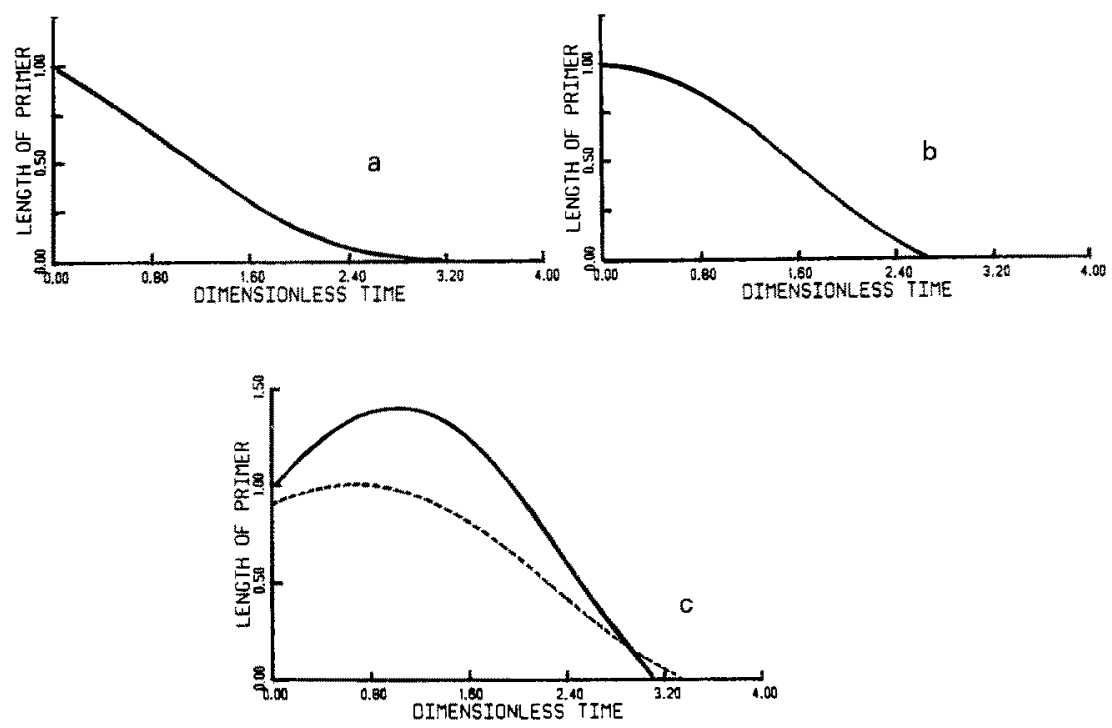

Fig. 7. Function $p_{v}(t)$ of Problem 1. 
$180^{\circ}$. The dimensionless final times are 3.071 and 2.684 ; the dimensionless characteristic velocities are 0.07139 and 0.05719 ; for an initial interceptor orbit with an altitude of $2000 \mathrm{~km}$, they translate into 62.14 minutes, 54.31 minutes, and $0.493 \mathrm{~km} / \mathrm{sec}, 0.394 \mathrm{~km} / \mathrm{sec}$, respectively, while the period of the inner orbit is $P=90.97$ minutes. But in the case $\omega=50^{\circ}$, the solution is not optimal. By the analysis in Section 4, we know that the behavior of $p_{V}(t)$ plotted in solid line in Fig. 7(c) suggests a coasting arc prior to the application of the impulse. To gain more insight, we look at the case where $\omega=34.78^{\circ}$. This lead angle is special, in the sense that a tangential retrograde impulse is optimal and the transfer angle $\Delta$ is exactly $\pi$, i.e., a Hohmann-type transfer. This special lead angle is given by

$$
\omega^{*}=\pi\left\{\sqrt{ }\left[(1 / 8)\left(1+r_{0} / r_{f}\right)^{3}\right]-1\right\} .
$$

When the initial $\omega$ is greater than $\omega^{*}$, the optimal strategy for the interceptor is to coast on the initial orbit until $\omega^{*}$ is formed due to relative motion, then launch. The coast time is computed from

$$
t_{1}=\left(\omega-\omega^{*}\right) / \Omega,
$$

where $\Omega$ is the relative angular speed,

$$
\Omega=\sqrt{\left(\mu / r_{f}^{3}\right)}-\sqrt{\left(\mu / r_{0}^{3}\right)} \text {. }
$$

After such a coast arc is added, $p_{V}(t)$ for $\omega=50^{\circ}$ is shown in Fig. 7(c) in dashed line. The optimal characteristic velocity is the same as in the case where $\omega=\omega^{*}=34.78^{\circ}$; the final time is 67.83 minutes; and the coast time is $t_{1}=13.52$ minutes.

Taking into account the constraint (38) and the fact that the Hohmanntype transfer requires $\Delta=\pi$, we can easily have the range of the initial lead angle $\omega$ within which the Hohmann-type optimal interception is possible,

$$
\omega^{*} \leq \omega \leq \pi\left\{\sqrt{ }\left[(1 / 8)(1+1 / n)^{3}\right]-2 / \sqrt{n^{3}}+1\right\},
$$

where $n$ is the ratio

$$
n=r_{0} / r_{f} \text {. }
$$

Whenever $\omega$ is within the range given by (43), the optimal characteristic velocity is the same,

$$
\Delta V=\sqrt{\left(\mu / r_{0}\right)}-\sqrt{ }\left[2 \mu r_{f} /\left(r_{0}\left(r_{0}+r_{f}\right)\right)\right] .
$$

When $0 \leq \omega<\omega^{*}$, we find that the initial one-impulse solution is always optimal, and always $t_{f}<P$. When $\omega$ exceeds the upper bound in (43) and is less than about $127^{\circ}$, we find that an initial coast is still needed, but of course is not given by (42) and the impulse is no longer tangential. Moreover, 
$t_{f}^{*}=P$; thus, condition (20) applies instead of (19). When $\omega$ is larger than about $127^{\circ}$, no initial coast is optimal, and $t_{f}^{*}=P$.

The dimensionless optimal characteristic velocity for different $\omega$ ranging from $0^{\circ}$ to $180^{\circ}$ is plotted in Fig. 8. It is seen that $\Delta V$ depends on the lead angle $\omega$ and can be prohibitive for large $\omega$. This dependence is due to the constraint (38). If $t_{f}$ is free, the interceptor can always stay on the initial orbit and launch when $\omega^{*}$ is formed, no matter what the initial configuration at $t_{0}$ is. The Hohmann-type interception is then performed, and the characteristic velocity is always the same, only depending on $n$. The launch time $0 \leq t_{1}$ is explicitly given by

$$
t_{1}= \begin{cases}\left(\omega_{0}-\omega^{*}\right) / \Omega, & \text { if } \omega_{0} \geq \omega^{*}, \\ \left(2 \pi+\left(\omega_{0}-\omega^{*}\right)\right) / \Omega, & \text { if } \omega_{0}<\omega^{*},\end{cases}
$$

where $\omega_{0}$ is the initial lead angle and $\omega^{*}$ is defined in (41). It is a simple exercise to show that the Hohmann transfer satisfies the conditions

$$
\begin{aligned}
& \mathbf{p}_{r}\left(t_{1}\right) \mathbf{p}_{V}\left(t_{1}\right)=0, \\
& \mathbf{p}_{r}\left(t_{f}\right)\left(\mathbf{V}_{T_{f}}-\mathbf{V}_{f}\right)=0 .
\end{aligned}
$$

For Hohmann transfer, $\mathbf{V}_{f}$ is parallel to $\mathbf{V}_{T f}$. Then, (48) is equivalent to

$$
H_{f}^{*}=\mathbf{p}_{r f} \mathbf{V}_{f}=0,
$$

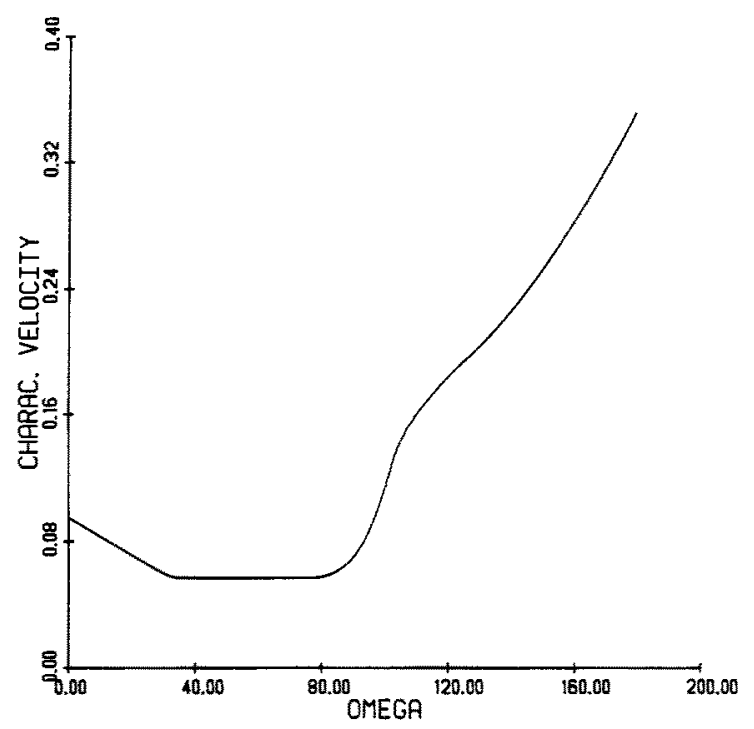

Fig. 8. Optimal characteristic velocity of Problem 1. 
hence $C=0$. If furthermore,

$$
\begin{aligned}
& A=0, \\
& B=(1-e) / 4 e, \\
& D=-(1+e)^{2}(1-e) / 4 e,
\end{aligned}
$$

where

$$
e=(n-1) /(n+1)
$$

then since $\theta_{0}=180^{\circ}$ and $\theta_{f}=0^{\circ}$ on the transfer orbit, we see that $p_{V}\left(t_{f}\right)=0$ and (47) holds. In addition, $S\left(t_{1}\right)=0$ and $T\left(t_{1}\right)=-1$, which is the characteristic of Hohmann transfer. But since $C=0$, we see from (26) that $N=0$; hence,

$$
2 S \tan (\Delta / 2)=\left(p / r_{0}\right) T=-p / r_{0} .
$$

From (27)

$$
\begin{aligned}
& A=-\left(p / r_{0}\right) \sin \theta_{f} /\left[4 \sin ^{2}(\Delta / 2)\right], \\
& B=\left(p / r_{0}\right) \cos \theta_{f} /\left[4 e \sin ^{2}(\Delta / 2)\right], \\
& D=-\left(p / r_{0}\right)\left[2 e+\left(1+e^{2}\right) \cos \theta_{f}\right] /\left[4 e \sin ^{2}(\Delta / 2)\right],
\end{aligned}
$$

Let $\Delta=180^{\circ}, \theta_{f}=0^{\circ}$, and $p / r_{0}=1-e$. We have (49).

Problem 2. When either or both orbits of the interceptor and the target are elliptic, the basic technique and analysis remain applicable though there may be no explicit relations like in the case of two circular orbits. If the intercept time is constrained by (38), the optimal solution is also expected to show dependence on the initial configuration.

Let us consider the case where the interceptor is still in a circular orbit defined by $r_{0}=1$, but the target is on an inner coplanar elliptic orbit with eccentricity $e_{T}$ and semilatus rectum $p_{T}$ (Fig. 9). It should be noticed that, although the orbits are well defined geometrically by the quantities $r_{0}, e_{T}$, and $p_{T}$, the motion with respect to the time on these orbits can be arbitrary. We assume that these motions are known. That is, at $t_{0}$, let $\eta$ be the lead angle of the interceptor with respect to the perigee of the target orbit; let $\omega$ be the lag angle of the target with respect to the perigee. Both $\eta$ and $\omega$ are known. For a given $\eta$, different $\omega$ represents different initial configuration.

As for numerical example, we consider a target orbit such that $e_{T}=0.2$, $p_{T}=0.6$, and select the time $t_{0}$ such that $\eta=30^{\circ}$. The angle $\omega$ is taken as a varying parameter. The method of solution is similar to that described in Problem 1 , with the flight time $t_{f}-t_{0}$ for a transfer angle $\Delta$ evaluated by Kepler's equation along the target orbit, instead of (40). 


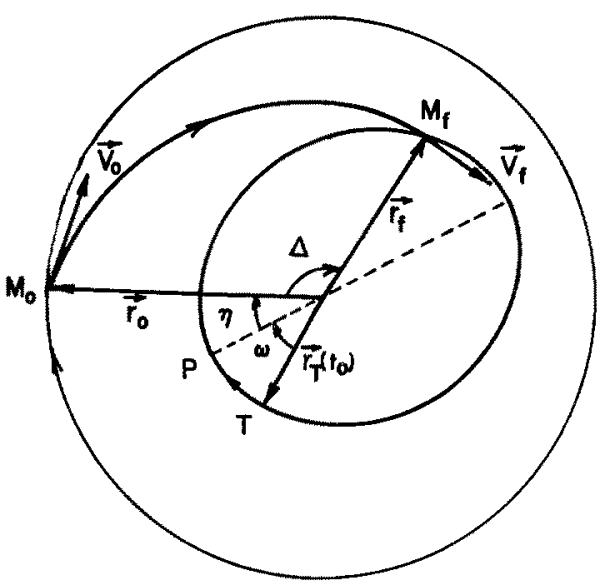

Fig. 9. Geometry of interception for Problem 2.

The verification of the function $p_{V}(t)$ against the discussion in Section 4 reveals that, for a given $\eta$, there exists an $\omega_{c}$, in our case $\omega_{c}=101.008^{\circ}$, such that, when $\omega \leq \omega_{c}$, the initial one-impulse solution is optimal. A coasting arc is needed for optimality when $\omega>\omega_{c}$. Furthermore, in our case, for $148.4^{\circ} \leq \omega<176.6^{\circ}, t_{f}^{*}=P=3.1046$ with a coasting prior to the impulse. For $\omega>176.6^{\circ}, t_{f}^{*}=P$ without coasting. Figure 10 gives the optimal

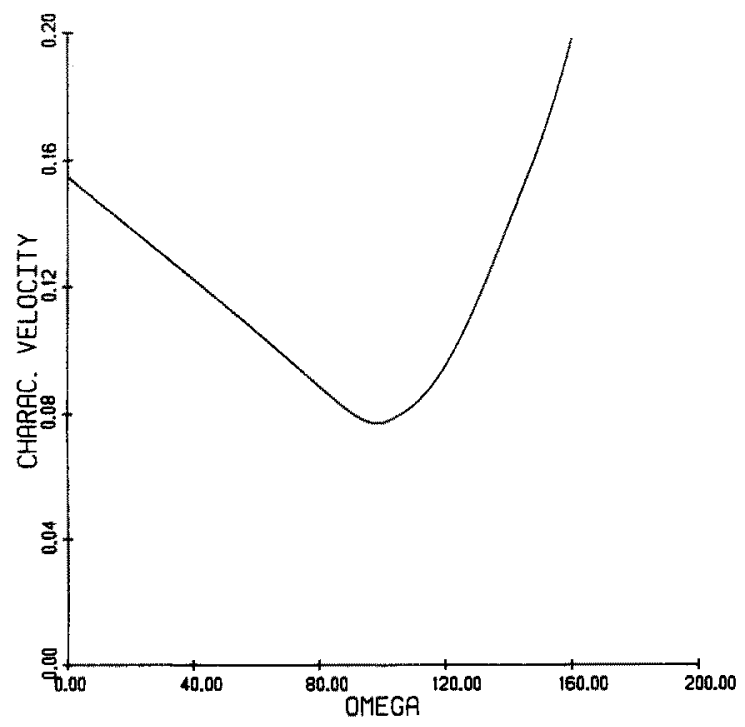

Fig. 10. Optimal characteristic velocity of Problem 2. 
$\Delta V$ vs $\omega$ for $0^{\circ} \leq \omega \leq 160^{\circ}$. It should be noted that, in the present situation, the optimal characteristic velocity shows stronger dependence on $\omega$ as in Fig. 10, unlike in Problem 1 where $\omega$ in a certain range yields the same $\Delta V$. There exists an overall optimal $\Delta V$ corresponding to $\omega^{*}<\omega_{c}$, in our case $\omega^{*}=98.437^{\circ}$. The special aspect of the optimal solution for $\omega^{*}$ is that the optimal transfer trajectory intersects tangentially the target orbit at the intercept point. We shall show that this particular trajectory is also the minimum fuel transfer trajectory from the position $\mathbf{r}_{0}\left(t_{0}\right)$ to the target orbit without considering interception.

Because of the tangency of the two orbits, $V_{T f}$ is parallel to $\mathbf{V}_{f}$. The transversality condition (19) is equivalent to

$$
H_{f}^{*}=\mathbf{p}_{r f} \mathbf{V}_{f}=0 .
$$

Hence, $C=0$ and $N=0$. From (26), we have

$$
\left(p / r_{0}\right) T+S\left[e \sin \theta_{0}-2 \tan (\Delta / 2)\right]=0,
$$

where $S$ and $T$ are the components of $\mathbf{p}_{V}\left(t_{0}\right)$ which are proportional to

$$
\begin{aligned}
& \left(1+e \cos \theta_{0}\right)^{2}\left[1-1 / \sqrt{ }\left(1+e \cos \theta_{0}\right)\right] \\
& =e \sin \theta_{0}\left[2 \tan (\Delta / 2)-e \sin \theta_{0}\right] .
\end{aligned}
$$

In deriving (51), we have used the polar equation,

$$
p / r_{0}=1+e \cos \theta_{0},
$$

to eliminate $p$. Next, by writing the condition for collinearity of the two vectors

$$
\begin{aligned}
& \mathbf{V}_{f}=\left(\sqrt{(\mu / p)} e \sin \theta_{f}, \sqrt{(\mu p)} / r_{f}\right), \\
& \mathbf{V}_{r f}=\left(\sqrt{\left(\mu / p_{T}\right)} e_{T} \sin (\eta+\Delta), \sqrt{\left(\mu p_{T}\right)} / r_{f}\right),
\end{aligned}
$$

we have

$$
\left(r_{0} / p_{T}\right)\left(1+e \cos \theta_{0}\right) e_{T} \sin (\eta+\Delta)=e \sin \left(\theta_{0}+\Delta\right) .
$$

Finally, we express the equality of the radii on the two orbits at $t_{f}$ as

$$
\left(r_{0} / p_{T}\right)\left(1+e \cos \theta_{0}\right)\left[1+e_{T} \cos (\eta+\Delta)\right]=1+e \cos \left(\theta_{0}+\Delta\right) .
$$

The three equations (51), (52), and (53) can be solved for the unknowns $e, \theta_{0}$, and $\Delta^{*}$. The angle $\omega^{*}$ can then be deduced from the Kepler equation.

The problem of finding a minimum fuel transfer orbit from a given position with one impulse to a given orbit is in the area of parametric optimization. We consider the problem of minimum fuel transfer from the position $\mathbf{r}_{0}\left(t_{0}\right)$ to the target orbit by following the hodograph theory presented in Ref. 7 . We look at the condition required for the velocity $V\left(t_{0}\right)$ after 
the impulse for leading the transfer trajectory to a point on the target orbit at the down range angle $\Delta$ with radius $r_{f}$. Let $x$ and $y$ be the components of the normalized velocity $V\left(t_{0}\right) / \sqrt{\left(\mu / r_{0}\right)}$ on the $M S T$ system. On the transfer orbit with eccentricity $e$, semilatus rectum $p$, and true anomaly $\theta$, we have

$$
\begin{aligned}
& x=\sqrt{\left(r_{0} / p\right)} e \sin \theta_{0}=e \sin \theta_{0} / \sqrt{ }\left(1+e \cos \theta_{0}\right), \\
& y=\sqrt{\left(p / r_{0}\right)}=\sqrt{ }\left(1+e \cos \theta_{0}\right) .
\end{aligned}
$$

At the final point,

$$
y_{f}=\sqrt{\left(p r_{0}\right)} / r_{f}=\sqrt{\left(r_{0} / p\right)}\left[1+e \cos \left(\theta_{0}+\Delta\right)\right] .
$$

Let

$$
n=r_{0} / r_{f}
$$

From (54)-(56), we have the equation for $x$ and $y$ in the MST system,

$$
(n-\cos \Delta) y^{2}+x y \sin \Delta-(1-\cos \Delta)=0 .
$$

Equation (57) shows that the tip of the velocity $\mathbf{V}\left(t_{0}\right)$ must be on a hyperbola with asymptotes $M S$ and $M M_{f}$ (Fig. 11). Since the initial normalized velocity has the components $(0,1)$, the minimum $\Delta V$ corresponds to the shortest distance from this point to the hyperbola (57) for prescribed $r_{f}$ and $\Delta$. When $r_{f}$ varies as a function of $\Delta$ (Fig. 9), we have a family of hyperbolas defined by

$$
\begin{aligned}
f(x, y, \Delta) & =\left\{\left(r_{0} / p_{T}\right)\left[1+e_{T} \cos (\eta+\Delta)\right]-\cos \Delta\right\} y^{2} \\
& +x y \sin \Delta-(1-\cos \Delta)=0 .
\end{aligned}
$$

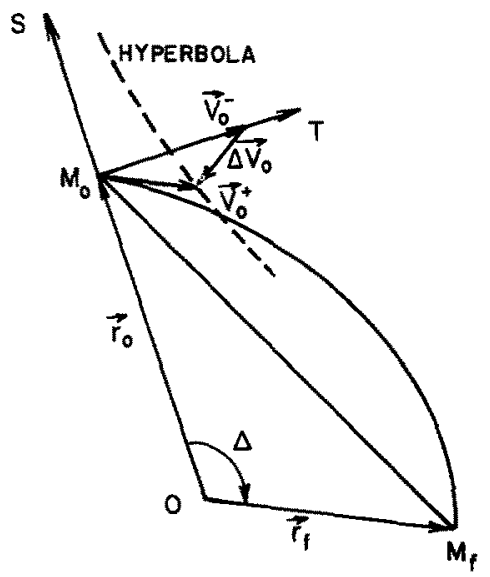

Fig. 11. Hodograph of $\mathbf{V}_{0}$. 
This family of hyperbolas has an envelope, which is obtained by eliminating $\Delta$ between (58) and equation $\partial f / \partial \Delta=0$,

$$
\left[\sin \Delta-\left(r_{0} / p_{T}\right) e_{T} \sin (\eta+\Delta)\right] y^{2}+x y \cos \Delta-\sin \Delta=0 .
$$

After elimination of $\Delta$ between (58) and (59), we arrive at

$$
\alpha y^{2}+x^{2}+2 \beta x y+\gamma=0,
$$

where

$$
\begin{aligned}
& \alpha=1+\left(r_{0} / p_{T}\right)^{2}\left(e_{T}^{2}-1\right)-2\left(r_{0} / p_{T}\right) e_{T} \cos \eta, \\
& \beta=-\left(r_{0} / p_{T}\right) e_{T} \sin \eta, \\
& \gamma=2\left[\left(r_{0} / p_{T}\right)\left(1+e_{T} \cos \eta\right)-1\right] .
\end{aligned}
$$

Since $\beta^{2}-\alpha>0$, the curve (60) is also a hyperbola. The terminus of the optimal velocity $\mathbf{V}\left(t_{0}\right)$ must be on this envelope. The shortest distance from the point $(0,1)$ to $(60)$ is obtained by solving $(60)$ and the equation for orthogonality,

$$
x /(x+\beta y)=(y-1) /(\alpha y+\beta x) .
$$

If we use an auxiliary variable $z$ defined by

$$
z=x / y=e \sin \theta_{0} /\left(1+e \cos \theta_{0}\right),
$$

by combining (60), (62), and (63), we obtain a quartic equation in $z$,

$$
A_{0} z^{4}+A_{1} z^{3}+A_{2} z^{2}+A_{3} z+A_{4}=0,
$$

where

$$
\begin{aligned}
& A_{0}=1+\gamma \beta^{2}, \\
& A_{1}=4 \beta-2 \beta \gamma(1-\alpha), \\
& A_{2}=\alpha+5 \beta^{2}+\gamma\left[(1-\alpha)^{2}-2 \beta^{2}\right], \\
& A_{3}=2 \alpha \beta+2 \beta^{3}+2 \beta \gamma(1-\alpha), \\
& A_{4}=\beta^{2}(\alpha+\gamma) .
\end{aligned}
$$

Upon solving (64), the components of the optimal $\mathbf{V}\left(t_{0}\right)$ are

$$
\begin{aligned}
& x=-(z+\beta) z /\left[\beta z^{2}+(\alpha-1) z-\beta\right], \\
& y=-(z+\beta) /\left[\beta z^{2}+(\alpha-1) z-\beta\right] .
\end{aligned}
$$

From (59) and with the aid of (60), (61), and (63), the optimal transfer angle is

$$
\tan (\Delta / 2)=-\gamma / 2(z+\beta) .
$$


To compare the above resuits with the results from optimal interception, we first notice that using definition (54) to expand (53) leads to (58). Likewise, (52) directly yields (59), consequently (60). Finally, using (51) and (52) to eliminate $\Delta$, after some algebraic manipulation, the quartic equation (64) is recovered.

We conclude this section by providing some explicit equations for computing the critical values $\omega_{c}$ where an initial coasting arc starts to appear. This happens when at the time of the impulse

$$
S \dot{S}+T \dot{T}=0 .
$$

Using this relation and noticing that, in this case,

$$
H_{0}^{*-}=H_{f}^{*}=-\mu \mathrm{Ce} / p^{2},
$$

we have a condition,

$$
\mu\left(\mathrm{Ce} / \mathrm{p}^{2}\right)=\sqrt{\left(\mu / r_{0}\right)} \dot{T}+\left(\mu / r_{0}^{2}\right) S .
$$

Making this equation explicit and simplifying, we have the relation

$$
2\left(p / r_{0}\right)^{3 / 2} \sin \Delta+\left(p / r_{0}\right)^{3} K-Q \sin \Delta=0,
$$

where

$$
\begin{aligned}
K & =I_{1}\left(\tau_{0}\right) \sin \theta_{f}-I_{1}\left(\tau_{f}\right) \sin \theta_{0} \\
& =\left[1 /\left(1-e^{2}\right)\right]\left[-\sin \Delta+2 e\left(r_{0} / p\right) \sin \theta_{f}-2 e\left(r_{f} / p\right) \sin \theta_{0}\right. \\
& \left.+3 \sqrt{\left(\mu / p^{3}\right)} \Delta t e^{2} \sin \theta_{0} \sin \theta_{f}\right], \\
Q & =1+e^{2}+2 e \cos \theta_{0}-2 e \sin \theta_{0} \tan (\Delta / 2) .
\end{aligned}
$$

In deriving these equations, we have used the simplification of an initial circular orbit. On the other hand, if we write the transversality condition (39), we obtain explicitly

$$
\begin{aligned}
& \left(p / r_{f}\right)^{2}\left[\left(p / r_{0}\right)^{3 / 2} K+\sin \Delta\right] \\
& \times\left[e \sqrt{\left(p_{T} / p\right)} \sin \theta_{f}-\sqrt{\left(p / p_{T}\right)} e_{T} \sin (\eta+\Delta)\right] \\
& =\left[Q-\left(p / r_{0}\right)^{3 / 2}\right]\left[e \sin \theta_{f}-\sqrt{\left(p / p_{T}\right)} e_{T} \sin (\eta+\Delta)\right] \sin \Delta .
\end{aligned}
$$

This equation is the general, time-free transversality condition for interception from initial circular orbit. In the case of $\omega=\omega_{c}$, Eq. (70) can be used to eliminate $K$, and we have the simple relation

$$
\begin{aligned}
& \left(p / r_{f}\right)^{2}\left[e \sqrt{\left(p_{T} / p\right)} \sin \theta_{f}-\sqrt{\left(p / p_{T}\right)} e_{T} \sin (\eta+\Delta)\right] \\
& =\left(p / r_{0}\right)^{3 / 2}\left[e \sin \theta_{f}-\sqrt{\left(p / p_{T}\right)} e_{T} \sin (\eta+\Delta)\right] .
\end{aligned}
$$


Now, if we use Eqs. (70) and (73), with $\theta_{f}=\theta_{0}+\Delta$ and the time of flight $\Delta t$ computed from Kepler's equation along the transfer orbit, and Eq. (53), we have three equations for the three unknowns $e, \theta_{0}$, and $\Delta$. The value $\omega_{c}$ then is computed from Kepler's equation along the target orbit. When $\omega=\omega_{1}>\omega_{c}$, the value $\eta=\eta_{1}$ which determines the required coasting arc becomes an additional unknown, but we can always solve the inverse problem by fixing $\eta_{1}$ for solving the three equations and then computing the corresponding critical $\omega$ for adjustment of $\eta_{1}$.

As special cases, we first notice that, for tangential interception, Eq. (52) applies and (72) is reduced to $Q=\left(p / r_{0}\right)^{3 / 2}$, which is precisely Eq. (51). For both tangential interception and $\omega=\omega_{c}$, Eq. (73) leads to $\sin \theta_{f}=0$. To satisfy all necessary conditions, we must have $\sin \theta_{0}=0, \sin \eta=0$. The transfer is of the Hohmann type.

Finally, we notice that, if the target orbit is circular, $e_{T}=0$ and the lead angle is irrelevant. Ruling out the very rare case of non-Hohmann type transfer where $e \sin \theta_{f}=0$, we obtain from Eq. (72)

$$
\left(p / r_{f}\right)^{3 / 2}\left[\left(p / r_{0}\right)^{3 / 2} K+\sin \Delta\right]=\left[Q-\left(p / r_{0}\right)^{3 / 2}\right] \sin \Delta .
$$

Equation (74) is the general transversality used in Problem 1 when $t_{f}^{*}<P$.

\section{Interception at Hyperbolic Speed}

In this section, we consider a case of realistic importance when $\mathbf{r}_{T}(t)$ represents the motion of a ballistic missile. The time $t_{f}$ is then finite and is usually the time before the missile reaches its maximum altitude. The initial $t_{0}$ cannot be arbitrary, usually some time after the detection of the motion of a hostile missile. Thus, both $t_{0}$ and $t_{f}$ are specified and the intercept time $\Delta t=t_{f}-t_{0}$ will be considerably short.

The geometry of interception is shown in Fig. 12. The interceptor is in its initial circular orbit with radius $r_{0}=1$. No extra difficulty will be present if an elliptic orbit is assumed, except for more parameters involved. At $t_{f}$, the target is at the position defined by the polar coordinates $r_{f}, \delta$, and $\varphi$, with $\delta$ being the longitude and $\varphi$ the latitude as measured from the position of the interceptor at $t_{0}$. The initial orbital plane of the interceptor is taken as the reference plane. the inclination angle between the reference plane and the interceptor-target plane at $t_{0}$ is given by

$$
\tan i=\tan \varphi / \sin \delta,
$$

and the angular distance between the interceptor and the target is given by

$$
\cos \Delta=\cos \varphi \cos \delta \text {. }
$$




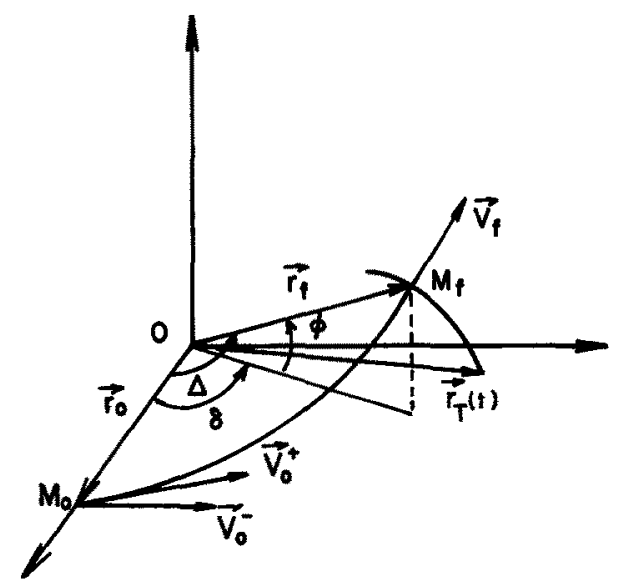

Fig. 12. Geometry of interception at prescribed $t_{f}$.

Again, the technique presented in section 4 is applied here. Because all end conditions are given, no transversality condition is involved. When $\Delta t$ is relatively short, the transfer trajectory is generally hyperbolic. The application of the technique of Section 4 shows that, in the hyperbolic region and the elliptic region where $\Delta t$ is not excessively long, the function $p_{V}(t)$ generated by an impulse applied at $t_{0}$ always falls into case (a) of Fig. 5 , so the initial one-impulse trajectory is optimal provided that the one-impulse transfer trajectory will not intersect the surface of the Earth, which is true for most of practical intercept situations. Only when $\Delta t$ is quite large do we have $p_{V}(t)$ belonging to case (b) of Fig. 5 where an initial coasting phase is required.

Figure 13 shows $\Delta V$ as a function of $\Delta t$ for a specified downrange longitude $\delta=45^{\circ}$, using the latitude $\varphi$ as parameter, with $20^{\circ}$ increment for an interception of altitude $r_{f}=0.95$. To have some physical feeling, an initial orbit of altitude $600 \mathrm{~km}$ is chosen; $r_{f}$ corresponds to an altitude of $251.23 \mathrm{~km}$. The intercept times range from 2 to 15 minutes.

We repeat the experiment with a value $r_{f}=1.05$, which corresponds to an altitude of $948.77 \mathrm{~km}$ for the same initial orbit. The results are illustrated in Fig. 14.

In each figure, we have plotted a dashed line separating the elliptic and hyperbolic interceptions. This is obtained by solving the equation for parabolic transfer,

$$
6 \sqrt{\mu} \Delta t=\left(r_{0}+r_{f}+c\right)^{3 / 2}-\left(r_{0}+r_{f}-c\right)^{3 / 2},
$$

where $c$ is the chord length,

$$
c^{2}=r_{0}^{2}+r_{f}^{2}-2 r_{0} r_{f} \cos \delta \cos \varphi
$$




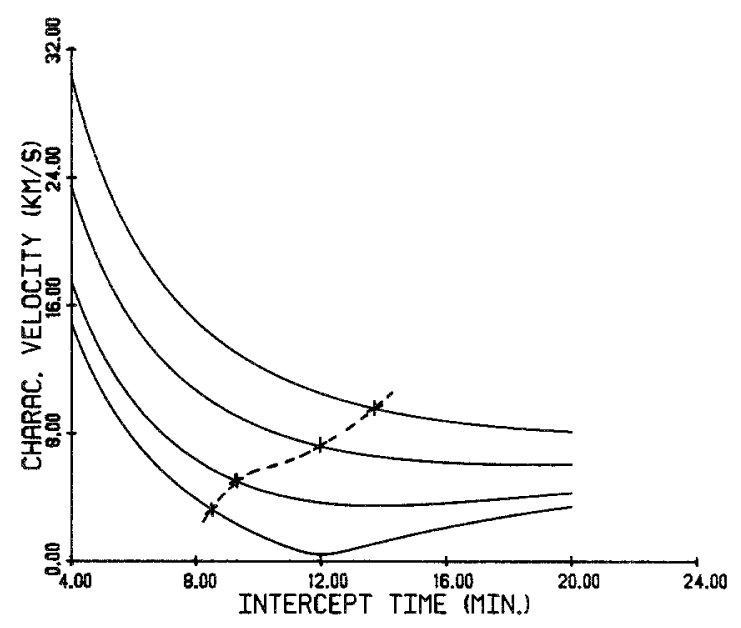

Fig. 13. Optimal characteristic velocity, $r_{f} / r_{0}=0.95$.

For each transfer time $\Delta t$ and downrange longitude $\delta$, it is obvious that, as we increase the latitude $\varphi$, the transfer angle $\Delta$ increases and the fuel consumption increases.

We notice that, for any given $\delta$ and $\varphi$, or in general for any prescribed transfer angle $\Delta$, there exists an optimal transfer time $\Delta t$ for overall minimum characteristic velocity. This particular transfer can be obtained as follows.

In the present formulation of the problem, allowing $\Delta t$ to vary is the same as fixing $\mathbf{r}_{0}$ and $\mathbf{r}_{f}$ and letting $t_{f}$ free. By the final remark in Section

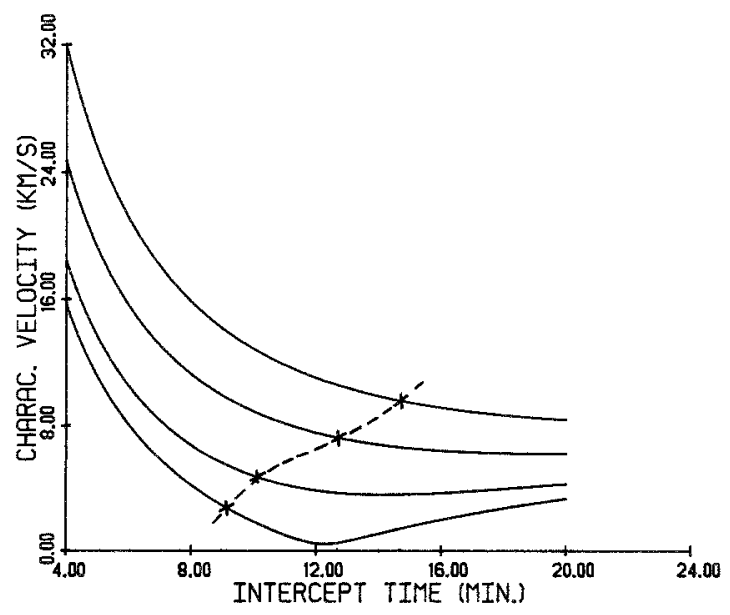

Fig. 14. Optimal characteristic velocity, $r_{f} / r_{0}=1.05$. 
3 , this leads to $H_{f}^{*}=0$, hence $C=0$. By $(50)$, we have

$$
\left(r_{0} / p\right)\left[2 \tan (\Delta / 2)-e \sin \theta_{0}\right]=T / S=v / u .
$$

It has been shown in Section 5 that, for a velocity $V_{0}$ to be such that the trajectory passes through the prescribed final point with radius $r_{f}$ and downrange $\Delta$, its normalized components $x$ and $y$ must satisfy the constraining relation (57). In general, let $\bar{x}$ and $\bar{y}$ be the components, along the $S$-axis and the $T$-axis, of the velocity $V_{0}^{-} / \sqrt{\mu / r_{0}}$ before the application of the impulse. We have

$$
\begin{aligned}
& \bar{x}=\sqrt{\left(r_{0} / p_{0}\right)} e_{0} \sin f_{0}, \\
& \bar{y}=\sqrt{\left(p_{0} / r_{0}\right)} \cos i .
\end{aligned}
$$

Notice that $\bar{x}$ and $\bar{y}$ are known quantities. Rewrite (79) by using definitions (21), (54), and (80),

$$
(y-\bar{y}) /(x-\bar{x})=\left(1 / y^{2}\right)[2 \tan (\Delta / 2)-x y] .
$$

The two equations (57) and (81) can be solved for $x$ and $y$. Explicitly, we solve for $x$ from (57),

$$
x=\left[(1-\cos \Delta)-(n-\cos \Delta) y^{2}\right] / y \sin \Delta .
$$

Upon substituting into (81), we have a quartic equation for $y$,

$$
A_{0} y^{4}+A_{1} y^{3}+A_{2} y^{2}+A_{3} y+A_{4}=0,
$$

where

$$
\begin{aligned}
& A_{0}=1+n^{2}-2 n \cos \Delta, \\
& A_{1}=\sin \Delta[(n-\cos \Delta) \tilde{x}-\bar{y} \sin \Delta], \\
& A_{2}=0, \\
& A_{3}=\bar{x}(1-\cos \Delta) \sin \Delta, \\
& A_{4}=-(1-\cos \Delta)^{2} .
\end{aligned}
$$

After solving for $x$ and $y$, we deduce the relevant elements of the transfer orbit from

$$
\begin{aligned}
& p / r_{0}=y^{2}=1+e \cos \theta_{0}, \\
& e \sin \theta_{0}=x y, \\
& \tan \theta_{0}=x y /\left(y^{2}-1\right), \quad \theta_{f}=\theta_{0}+\Delta, \\
& e^{2}=(x y)^{2}+\left(y^{2}-1\right)^{2}, \\
& a=r_{0} /\left[2-\left(x^{2}+y^{2}\right)\right] .
\end{aligned}
$$


The minimum overall characteristic velocity is computed from (21) and (22), where now

$$
\begin{aligned}
& u=\sqrt{\left(\mu / r_{0}\right)}(x-\bar{x}), \\
& v=\sqrt{\left(\mu / r_{0}\right)}(y-\bar{y}) .
\end{aligned}
$$

The optimal time of flight $\Delta t$ is obtained from Kepler's equation,

$$
\sqrt{\left(\mu / a^{3}\right) \Delta t}=\left(E_{f}-E_{0}\right)-e\left(\sin E_{f}-\sin E_{0}\right),
$$

where (32) is used to evaluate the eccentric anomalies $E_{0}$ and $E_{f}$. The computation using these explicit equations indeed gives the points of minimum $\Delta V$ in Figs. 13 and 14.

\section{Conclusions}

In this paper, we have presented the complete solution of the problem of interception with time constraint for an interceptor with high-thrust propulsion system. The necessary conditions and the transversality conditions for optimality were discussed. The method of solution amounts to first solving a set of equations to obtain the primer vector for an initial one-impulse solution. Then, based on the information provided by the primer vector, rules are established to search for the optimal solution if the initial one-impulse trajectory is not optimal. The approach is general, in the sense that it allows for solving a problem of three-dimensional interception with arbitrary motion for the target.

Several numerical examples are presented, including orbital interceptions and ballistic missile interception. Since impulsive thrust is assumed, whenever it is convenient, the results from optimal control theory are verified by parametric optimization using hodograph theory. In the important case of short-time interception of a ballistic missile, it is found that the intercept trajectory is usually hyperbolic and, for a minimum fuel trajectory, a single impulse is to be applied immediately at the acquisition time.

\section{References}

1. LAWDEN, D. F., Optimal Trajectories for Space Navigation, Butterworth, London, England, 1963.

2. Marec, J. P., Optimal Space Trajectories, Elsevier Scientific Publishing Company, Amsterdam, Holland, 1979.

3. VINH, N. X., Integration of the Primer Vector in a General Central Force Field, Journal of Optimization Theory and Applications, Vol. 9, No. 1, pp. 51-58, 1972.

4. LeItMann, G., The Calculus of Variations and Optimal Control: An Introduction, Plenum Press, New York, New York, 1981. 
5. Battin, R. J., and Vaughan, R. M., An Elegant Lambert Algorithm, Journal of Guidance, Control, and Dynamics, Vol. 7, No. 6, pp. 662-670, 1984.

6. LiOn, P. M., and HANDElman, M., The Primer Vector on Fixed-Time Impulsive Trajectories, AIAA Journal, Vol. 6, No. 1, pp. 127-132, 1968.

7. Vinh, N. X., Busemann, A., and Culp, R. D., Hypersonic and Planetary Entry Flight Mechanics, University of Michigan Press, Ann Arbor, Michigan, 1980. 